Virtual Mentor. February 2003, Volume 5, Number 2.

doi: 10.1001/virtualmentor.2003.5.2.ccas1-0302

Clinical Cases

\title{
Does Patient Autonomy Outweigh Duty to Treat?
}

\section{Patients can refuse medical treatment, but physicians often question the patients' ability to make a responsible decision in a time of medical need.}

\author{
Commentary by Catherine A. Marco, MD
}

Dr.Avery, a second-year emergency medicine resident, was on duty in a large urban hospital one night with 2 other residents, when a police officer escorted in a young man. The officer had found Scott Daley semi-conscious and with a cut on his head. When questioned by the officer, Mr. Daley had responded quickly, telling the officer that he had fallen. He then stood up and walked without difficulty. To be on the safe side, the officer decided that Mr. Daley should receive medical attention. Mr. Daley agreed.

Upon physical examination, Dr. Avery documented a minor laceration on Mr. Daley's forehead and some significant occipital swelling and tenderness. He appeared slightly intoxicated but was alert and aware. He understood the physician's questions and answered basic questions, such as his name, the location, and the date. However, he did not cooperate with more detailed questioning. He said that he had hit his head on the sidewalk and that it hurt. His pupils were equally round and reactive to light. There was no obvious evidence of intracranial bleeding, but Dr. Avery said she wanted to do a CT scan to be certain there was no skull fracture or intracranial bleeding.

At this suggestion, Mr. Daley became argumentative. He did not want any tests and did not want to spend any more time in the emergency room. He said he was fine. "Just give me some aspirin or something for the headache, and I'm outta here," he said. Because of the signs of head trauma and her difficulty in completing a detailed mental status exam, Dr. Avery did not want to let Mr. Daley leave the hospital before ruling out significant internal head injury. She attempted to explain the gravity of the situation, and risks of leaving without allowing her to complete diagnostic tests. The patient seemed inattentive and refused to cooperate with further questioning.

After reasoning with the patient for several minutes to no avail, Dr. Avery asked the other residents to help her convince the patient. They were equally unsuccessful, and the patient became increasingly agitated. He said that they were "ganging up on" him. He got up and started to leave. Dr. Avery could, of course, allow the patient to leave against medical advice. Given the possibility that he could have a life-threatening head injury, though, she contemplated whether to restrain him and complete the diagnostic work-up against his will.

\section{Commentary}

This interesting case depicts a scenario commonly encountered in emergency medicine. The fundamental question in this case is whether the patient possesses appropriate decisional capacity to make an important choice regarding refusal of medical care.

Some erroneously believe that decisional capacity (sometimes referred to as "competence") is an all-or-nothing phenomenon-either the patient has the capacity to consent to medical treatment or he does not. Unfortunately, the concept is not so simple.

Decisional capacity is based on the patient's ability to understand the choices, to deliberate about those choices, and to articulate his choice. Decisional capacity is dynamic — that is, a patient who had appropriate decisional capacity 
yesterday may not have it today. There are numerous reversible causes of impaired decisional capacity, including intoxication, hypoxia, sedation, stress, and many others. Every effort should be made to reverse potential impairments in capacity, to assure that the patient is making the most rational, autonomous choice. Level of needed capacity may also be decision-specific. For example, the physician may have a lower threshold for allowing a patient to refuse suturing a small laceration, than for allowing a patient to refuse admission for a myocardial infarction.

The medical evaluation of capacity can be challenging. On some level, we assess the capacity of every patient we see. For most patients, we presume that if they are able to give a rational history, cooperate with the medical evaluation, and appear to understand the treatment recommendations, they possess appropriate decisional capacity. Impaired capacity comes into question most often when patients refuse recommended medical treatment.

To evaluate decisional capacity appropriately, all reversible threats to capacity should be addressed. Patient goals and values should be assessed. Alternatives and consequences should be discussed, and the patient should demonstrate understanding of these elements. Family members may be helpful in determining mental status as compared to baseline. Standardized tests, such as the Mini-Mental Status Examination, may be helpful.

Another common misconception is that a signature on an "against medical advice" (AMA) form is sufficient to allow a patient to leave. In fact, informed refusal is a process, not merely a signature on an AMA form. The process should consist of determination of decisional capacity, delivery of information, including risks of refusing treatment, and documentation of the process.

In this particular case, the physician must make a judgment regarding decisional capacity. It is challenging because the patient exhibits some elements of capacity - he is awake, alert, and answers some questions appropriately. However, there is also evidence of impaired capacity. The patient appears somewhat intoxicated and is somewhat uncooperative. He is unable (or unwilling) to cooperate with detailed questioning, which makes it impossible to ascertain whether he understands the risks of leaving against medical advice.

The significance of alcohol intoxication and its relationship to decisional capacity is controversial. Some believe that any intoxication renders a patient unable to make medical decisions. Others believe that intoxicated patients should be evaluated for appropriate capacity. In some cases, intoxicated patients are in fact able and willing to understand choices and the ramifications of those choices and to make an autonomous decision. A blood alcohol level is not necessary or sufficient to make a determination of appropriate decisional capacity. However, it may provide supporting evidence of impaired capacity. Certainly, alcohol intoxication is a "red flag," suggesting to the clinician possible impairment of capacity.

In cases where a complete evaluation of decisional capacity is not possible, it is reasonable to detain the patient until such an evaluation can be completed. Some options include explaining this simple fact to the patient. Often, when faced with the possibility of being physically or chemically restrained, the patient suddenly becomes more cooperative with answering questions. If the patient is able and willing to answer questions appropriately and understands the risks of leaving against medical advice, he should be allowed to leave. If he is not, he should be restrained until an appropriate evaluation of capacity can be completed.

Catherine A. Marco, MD, currently serves as chair of the American College of Emergency Physicians' Ethics Committee. She is also an associate professor at the Medical College of Ohio and an attending physician at St Vincent Mercy Medical Center, Toledo, Ohio.

The people and events in this case are fictional. Resemblance to real events or to names of people, living or dead, is entirely coincidental. The viewpoints expressed on this site are those of the authors and do not necessarily reflect the views and policies of the AMA.

(C) 2003 American Medical Association. All Rights Reserved. 